\title{
THE COUNTS OF RADIO SOURCES
}

\author{
M. S. LONGAIR \\ Mullard Radio Astronomy Observatory, Cavendish Laboratory, Cambridge, U.K.
}

\section{Introduction}

Following the advice of Professor Zel'dovich, I will begin with two platitudes - first, when we consider the implications of the counts of radio sources, we must look at all the counts of radio sources at all frequencies; second, when we attempt to evaluate the implications of the source counts, we should use proper cosmological models and not rely on the local Euclidean model.

I will cover three topics:

(i) What are the essential features of the source counts in the light of all the new data which have recently become available? I will present the counts made at the following frequencies - 178, 408, 1420, 2700, $5000 \mathrm{MHz}$

(ii) The important question of the isotropy of the distribution of radio sources on the sky.

(iii) I will describe three interpretations of the source counts, concentrating upon the evolutionary models in which it is assumed that the radio source population evolves strongly with cosmological epoch.

All the counts will be presented in differential form so that the numbers of sources $n$ counted in each flux density interval $\Delta S$ are statistically independent. The counts will be presented in the form $n / n_{0}$ where $n_{0}$ is the differential number of sources expected in a Euclidean universe - i.e. $n_{0} \propto\left(S^{-3 / 2}-(S+\Delta S)^{-3 / 2}\right)$. In a Euclidean universe in which sources were distributed uniformly, $n / n_{0}$ would be a constant for all flux densities. In real world models, however, the differential counts of sources depart significantly from the constant value, even at relatively small redshifts. This is illustrated in Figure 1 which is a theoretical plot of the expected behaviour of $n / n_{0}$ as a function of flux density for a single radio luminosity class $P_{0}$ of sources which have power-law spectra defined by $S \propto v^{-0.75}$ (i.e. the spectral index $\alpha=0.75$ ) and which are uniformly distributed throughout an Einstein-de Sitter world model. There is a simple analytic form for this case

$$
\begin{aligned}
\frac{n}{n_{0}} & =\frac{(1+z)^{-1.5(1+\alpha)}}{\left[(1+\alpha)(1+z)^{1 / 2}-\alpha\right]} \\
S & =\frac{P_{0}}{\frac{4 c^{2}}{H^{2}}\left(1-(1+z)^{-1 / 2}\right)^{2}(1+z)^{1+\alpha}}
\end{aligned}
$$

On the curve I have marked the redshift at which the source is observed and it can be seen that the curve deviates markedly from the Euclidean prediction at redshifts much 
log. (Flux density)

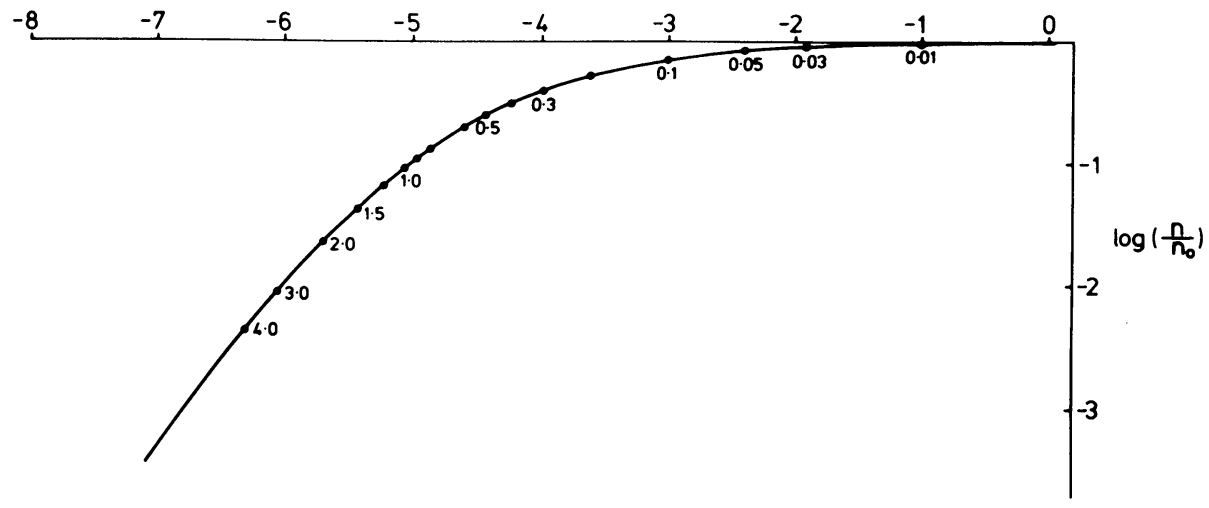

Fig. 1. The theoretical differential source count, expressed as $n / n_{0}$, for a single radio luminosity class of source having spectral index $\alpha=0.75$ in an Einstein-de Sitter world model $(\Omega=1 ; \Lambda=0)$. The redshift at which sources are observed is indicated on the curve.

smaller than 1. To obtain a more realistic estimate of the expected source counts for a uniform world model, this function must be convolved with the luminosity function of radio sources and this will be done in Section 4.

\section{Counts of Radio Sources}

\section{1. $178 \mathrm{MHz}$}

Figure 2 shows the Cambridge counts at $178 \mathrm{MHz}$ in differential form. These are derived from the $3 \mathrm{C}, 4 \mathrm{C}$ and North Polar surveys. In the case of the points derived from the $4 \mathrm{C}$ survey a new set of corrections has been derived to take account of con-

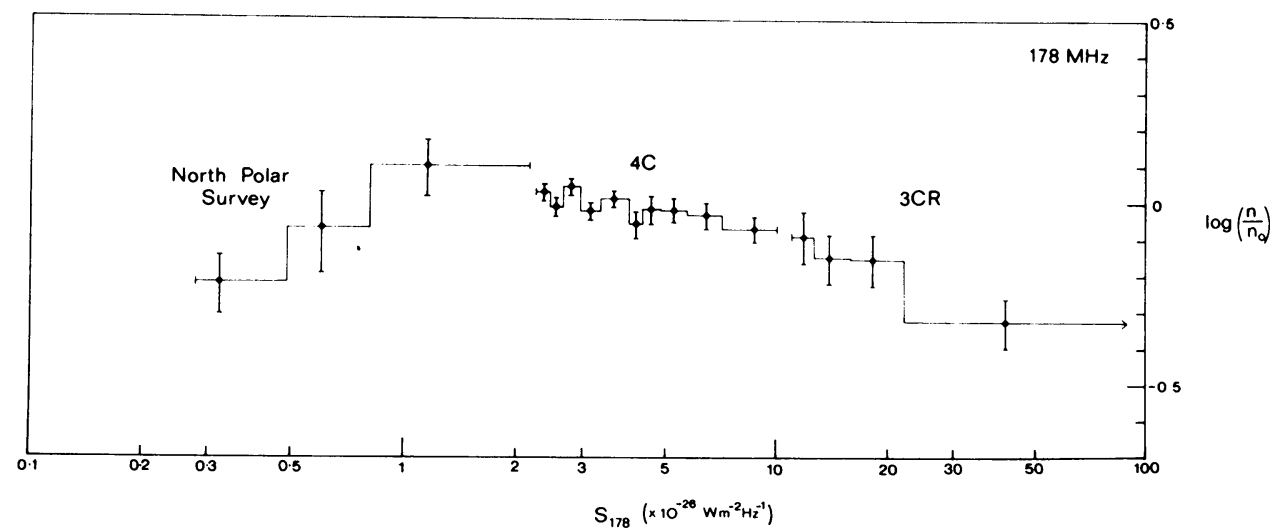

Fig. 2. The differential counts of radio sources at $178 \mathrm{MHz}$ derived from the $3 \mathrm{CR}, 4 \mathrm{C}$ and North Polar surveys (Bennett, 1962; Gower, 1966; Ryle and Neville, 1962). New corrections have been derived to account for underestimation of the flux densities of sources of large angular size in the $4 \mathrm{C}$ catalogue. The counts are normalised to 706 sources $\mathrm{sr}^{-1}$ having $S_{178} \geqslant 2 \times 10^{-26} \mathrm{~W} \mathrm{~m}^{-2} \mathrm{~Hz}^{-1}$. 
fusion, errors and underestimates in the numbers of sources of large angular size which may be missed because the survey was made with an interferometer. The last correction was derived from an analysis of further information which has become available on the fraction of sources of large angular size in the $4 \mathrm{C}$ catalogue, principally due to the work of Caswell and Crowther (1969). Details of this analysis will be presented in a forthcoming paper by Hooley, Pearson and myself.

It can be seen that the slope of the counts of $3 \mathrm{C}$ sources is steeper than the Euclidean count. A maximum likelihood estimate of the exponent of the integral counts, $\beta$, gives $\beta=1.9 \pm 0.1$ compared with the Euclidean value of 1.5. An important question is to what flux density this 'deficit' of bright sources extends. A literal interpretation of Figure 2 suggests that it extends to about $5 \times 10^{-26} \mathrm{~W} \mathrm{~m}^{-2} \mathrm{~Hz}^{-1}$. However, this must be treated with some caution because of statistical uncertainties and because of residual uncertainties in the angular diameter corrections.

The North Polar Survey suggests that the counts decrease below $1 \times 10^{-26} \mathrm{~W} \mathrm{~m}^{-2}$ $\mathrm{Hz}^{-1}$. Taken on their own, they cannot be considered strong evidence for convergence of the counts because of the limited statistics. However, Hewish's analysis of the record deflections of the $4 \mathrm{C}$ survey indicates that such a convergence must necessarily occur at about these flux densities (Hewish, 1961).

\section{2. $408 \mathrm{MHz}$}

Figure 3 shows the Cambridge counts of radio sources at $408 \mathrm{MHz}$ and the counts derived from the Parkes $408 \mathrm{MHz}$ survey as compiled by Ekers (1969). It can be seen that the Parkes and Cambridge counts are in excellent agreement at high flux densities and indicate the persistence of the deficit of sources at high flux densities as compared with the Euclidean prediction. The second important feature is the marked convergence of the counts at flux densities less than $0.5 \times 10^{-26} \mathrm{~W} \mathrm{~m}^{-2} \mathrm{~Hz}^{-1}$. This

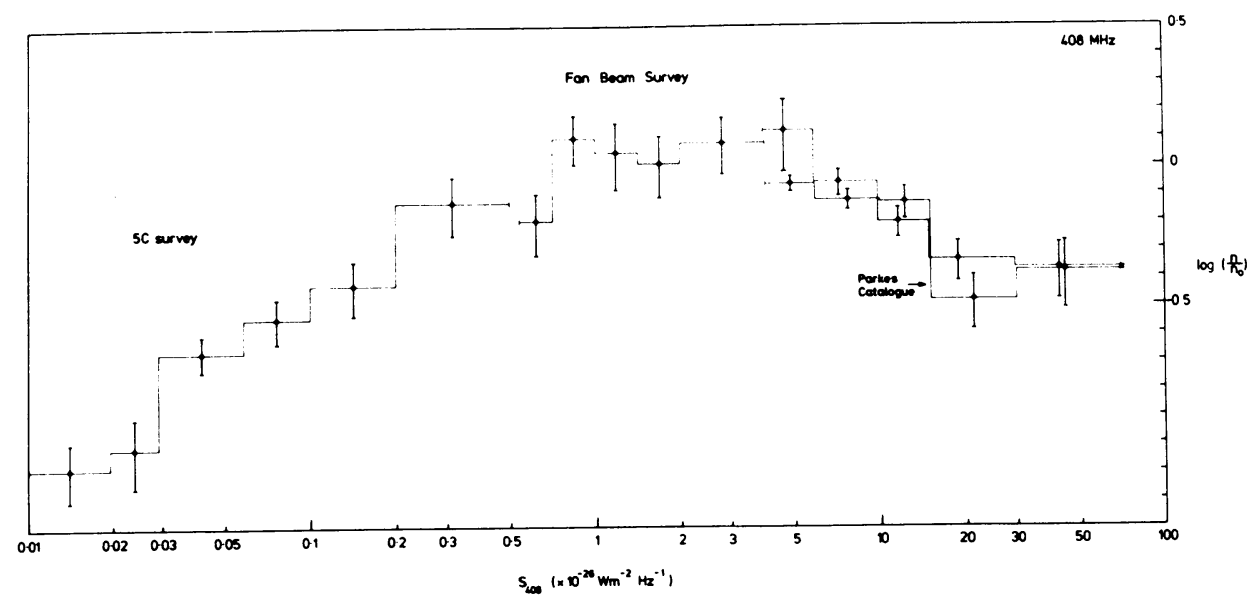

Fig. 3. The differential counts of radio sources at $408 \mathrm{MHz}$ (derived from the work of Pooley and Ryle, 1968). Also shown are counts derived from the Parkes catalogue (Ekers, 1969). The counts are normalised to 750 sources $\mathrm{sr}^{-1}$ having $S_{408} \geqslant 10^{-26} \mathrm{~W} \mathrm{~m}^{-2} \mathrm{~Hz}^{-1}$. 
convergence is found not only in the $5 \mathrm{C} 2$ survey which is presented here but also in the 5C3 and 5C4 surveys. The slope of the integral counts for flux densities less than $0.5 \times 10^{-26} \mathrm{~W} \mathrm{~m}^{-2} \mathrm{~Hz}^{-1}$ is 0.8 which deviates grossly from the Euclidean prediction.

More recently, the first results of the Molonglo surveys at $408 \mathrm{MHz}$ which will eventually cover the whole southern hemisphere have become available (Mills et al., 1973) and these counts are compared with the Cambridge counts in integral form in Figure 4. It can be seen that the agreement is excellent. It should be noted that for flux densities greater than $10 \times 10^{-26} \mathrm{~W} \mathrm{~m}^{-2} \mathrm{~Hz}^{-1}$ the counts have been derived by counting all sources on the celestial sphere away from the galactic plane (Robertson, 1973). The counts are shown in differential form in Figure 5.

\section{3. $1420 \mathrm{MHz}$}

In Figure $6 \mathrm{I}$ have collected together four surveys - the high flux density survey of Bridle et al. (1972), the GA survey of Davis (1974), the GB survey of Maslowski (1973a) and the first preliminary counts derived using the Westerbork synthesis telescope (Katgert et al., 1973). I have included no corrections for confusion or errors in the GB survey (this has now been done by Maslowski, 1973b).

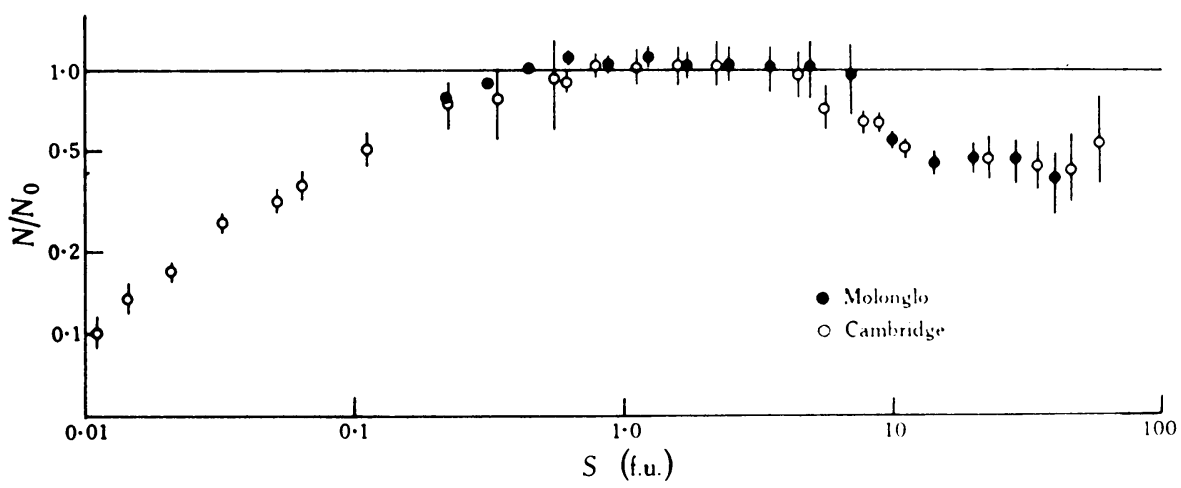

Fig. 4. Comparison of the integral source counts at $408 \mathrm{MHz}$ obtained from the Molonglo and Cambridge catalogues.

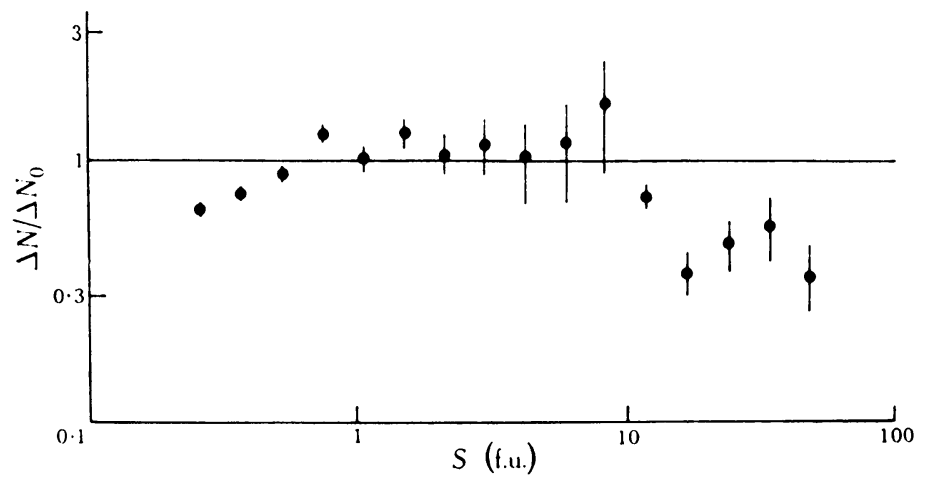

Fig. 5. The differential counts of radio sources at $408 \mathrm{MHz}$ from the Molonglo surveys and the whole sky compilation for $S_{408} \geqslant 10 \times 10^{-26} \mathrm{~W} \mathrm{~m}^{-2} \mathrm{~Hz}^{-1}$ (Mills et al., 1973; Robertson, 1973). 
It is immediately striking that the counts at $1420 \mathrm{MHz}$ have the same overall shape as at $408 \mathrm{MHz}$ showing an initial slope of 1.9 and convergence at low flux densities. There are now, however, some indications of anisotropies in the numbers of sources in different parts of the sky and this is illustrated in Figure 6 in the overlap region between the last three surveys listed above - i.e. in the range 3 to $0.3 \times 10^{-26}$

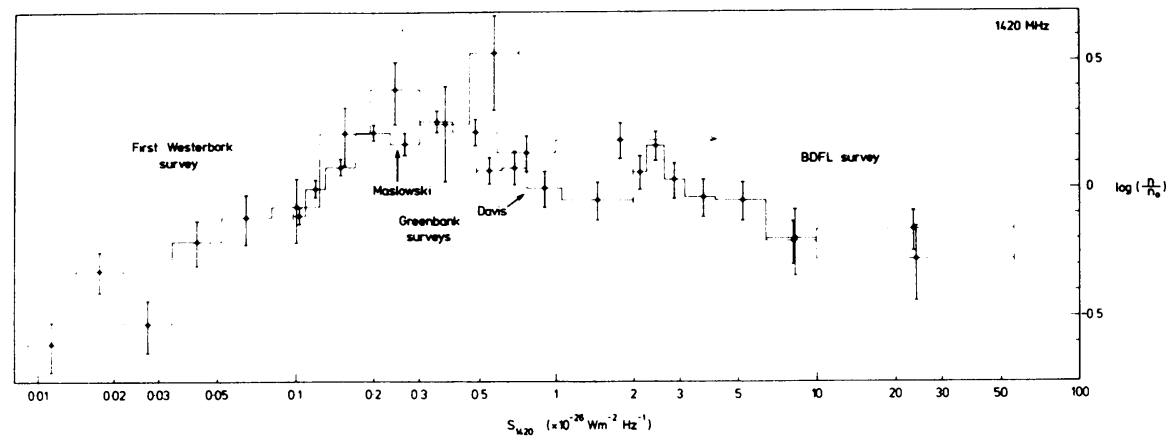

Fig. 6. The differential counts of radio sources at $1420 \mathrm{MHz}$. The counts are taken from the work of Bridle et al. (1972), Davis (1973), Katgert et al. (1973 - First Westerbork survey), and Maslowski (1973a). The counts are normalised to 150 sources $\mathrm{sr}^{-1}$ having $S_{1420} \geqslant 10^{-26} \mathrm{~W} \mathrm{~m}^{-2} \mathrm{~Hz}^{-1}$.

$\mathrm{W} \mathrm{m} \mathrm{m}^{-2} \mathrm{~Hz}^{-1}$. The GB survey of Maslowski has the largest statistics and a comparison of this survey with the Westerbork counts suggests that the differences in the numbers of sources are not statistically significant, particularly in view of the small numbers of sources counted by the Westerbork workers in the overlap region.

The difference between the GA and GB surveys looks more serious. The flux density scales of the surveys have been carefully checked and they are known to be correct. Maslowski estimates that the difference between the surveys is significant at the $98 \%$ confidence level (see also the report by Flin et al., 1974).

How far does this anisotropy invalidate the overall picture of the source counts? In my view, if this turns out to be a real effect, it is of a much smaller order of magnitude than the gross features of the counts which I consider to be the deficit of sources at high flux densities and the rapid convergence of the counts at low flux densities. This is illustrated quantitatively in Figure 6. However, it is of the greatest importance to investigate the nature and extent of these anisotropies since they may contain important information about the largest scale structures in the Universe at late epochs.

\section{4. $2700 \mathrm{MHz}$}

Figure 7 shows the counts derived from the Parkes $2700 \mathrm{MHz}$ survey (Bolton 1971). It is evident that they are much flatter than the counts at lower frequencies although the statistical error at high flux densities is quite large. At the present time, the Parkes survey is being completed and will eventually cover the whole southern sky plus the northern sky as far as $\delta=25^{\circ}$.

At high flux densities about 3 sr of sky have been surveyed by Porcas, Walsh and 


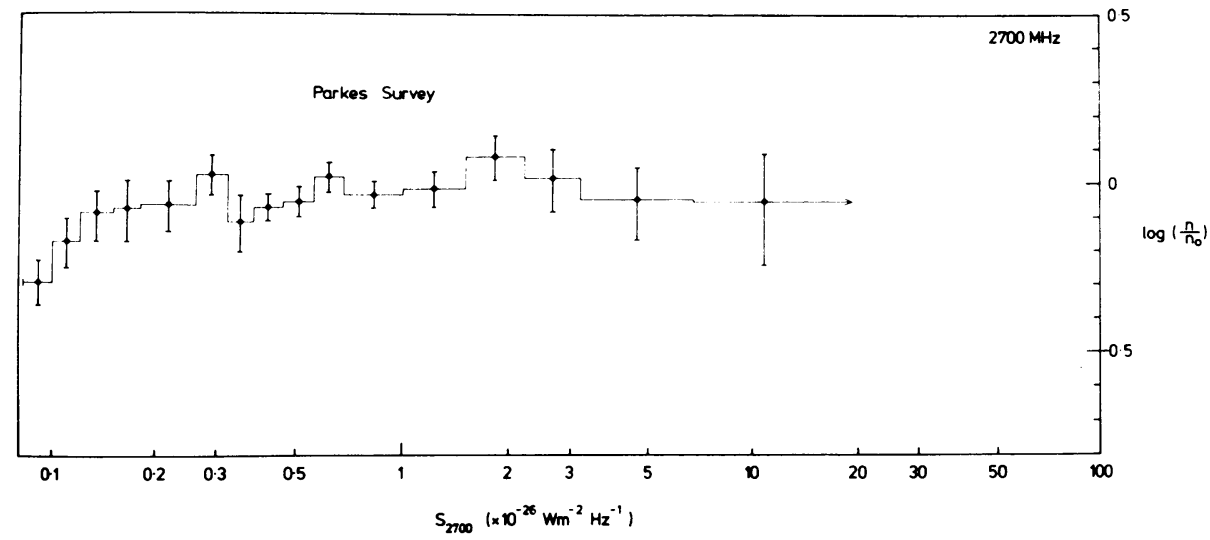

Fig. 7. The differential counts of radio sources at $2700 \mathrm{MHz}$ taken from the review by Bolton (1971). The counts are normalised to 100 sources $\mathrm{sr}^{-1}$ having $S_{2700} \geqslant 10^{-26} \mathrm{~W} \mathrm{~m}^{-2} \mathrm{~Hz}^{-1}$.

their colleagues at Jodrell Bank. They have constructed a complete sample of 120 sources in $3.8 \mathrm{sr}$ having flux densities greater than $1.8 \times 10^{-26} \mathrm{~W} \mathrm{~m}^{-2} \mathrm{~Hz}^{-1}$. Combining their results with part of the Parkes survey, they find a maximum likelihood slope of $1.74 \pm 0.15$ for flux densities greater than $1.5 \times 10^{-26} \mathrm{~W} \mathrm{~m}^{-2} \mathrm{~Hz}^{-1}$. Within the statistical uncertainties, this result is in agreement with the Parkes survey.

\section{5. $5000 \mathrm{MHz}$}

Figure 8 shows the latest results of the Greenbank surveys (see also the review by Pauliny-Toth and Kellermann in this volume, p. 111). There may be a deficit of sources at high flux densities but it is not of much significance in view of the limited statistics. An independent sample is available from the Parkes surveys in which flux densities are also measured at $5000 \mathrm{MHz}$. Shimmins and Bolton (1974a) have generated a sample of sources at $5000 \mathrm{MHz}$ which is complete to $0.58 \times 10^{-26} \mathrm{~W} \mathrm{~m}^{-2}$

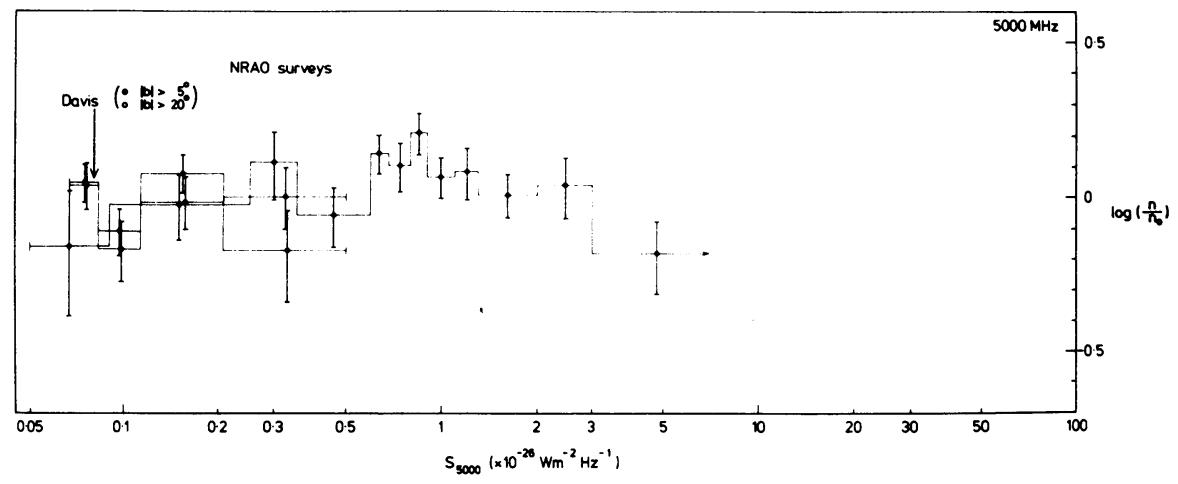

Fig. 8. The differential counts of radio sources at $5000 \mathrm{MHz}$ derived by Pauliny-Toth and Kellermann (1972) and by Davis (1971). The counts are normalised to 50 sources $\mathrm{sr}^{-1}$ having $S_{5000} \geqslant 10^{-26} \mathrm{~W} \mathrm{~m}^{-2} \mathrm{~Hz}^{-1}$. 
$\mathrm{Hz}^{-1}$. They find an integral source count which can be represented very well by

$$
N=58.0 S^{-1.50} \text {. }
$$

This result is in excellent agreement with the Greenbank surveys.

\subsection{SUMmary}

From this survey it can be concluded that:

(i) there is a significant deficit of sources at high flux densities at 178, 408 and $1420 \mathrm{MHz}$;

(ii) the deficit is not so evident at 2.7 and $5 \mathrm{GHz}$ although the observations would be consistent with a similar deficit to that observed at lower frequencies;

(iii) the counts converge rapidly at low flux densities.

The question of anisotropies in the distribution of sources will now be considered.

\section{Anisotropies}

In the spirit of my first platitude I mention first:

(i) The microwave background radiation. The evidence which is surveyed by Drs Boynton and Partridge in this volume gives us confidence that on the largest scale the Universe is remarkably isotropic.

(ii) At $178 \mathrm{MHz}$ we have intensively studied the isotropy of sources listed in the 4C catalogues. Holden (1966) has shown that there is no evidence for anisotropy on any scale greater than $0.5^{\circ}$ in the distribution of the $50004 \mathrm{C}$ sources on the celestial sphere. This result is confirmed on small scales by the nearest-neighbour analysis of Hinder and Branson (1969). Hughes and Longair (1967) investigated the isotropy of fainter sources by studying the distribution of record deflections $(P(D))$ throughout the region of the $4 \mathrm{C}$ survey. No evidence of anisotropy was found.

(iii) We have already described in Section 2 the differences between the GA and GB surveys which are significant at the $98 \%$ confidence level.

(iv) Yahil (1972) has claimed that the shape of the source counts differs in the northern and southern galactic hemispheres. This effect is only found in certain high frequency surveys and is significant at the 2 to $3 \sigma$ significance level. Pearson (1974) has repeated this test for the $3 \mathrm{C}$ and $4 \mathrm{C}$ surveys using the maximum likelihood estimate of the slope of the integral source counts as a measure of the shape of the counts. The result is shown in Table I as well as the result for the NRAO $5 \mathrm{GHz}$ survey (Pauliny-Toth and Kellermann, 1972). There is no evidence for any significant difference between the slopes of the counts in the northern and southern galactic hemispheres at low frequencies. If this is a real effect it may be related to the anisotropies discussed in (v) and (vi).

(v) The Greenbank GB survey suggests that there are significant differences in the spectral index distribution of radio sources in the 5C1 and 5C2 regions of sky (Maslowski, 1972 - see Figure 9). The correctness of the spectra is testified by the fact that for several of the sources observations are available at three frequencies, 408, 1420 
TABLE I

Slope of the integral source-counts in the northern and southern galactic hemispheres

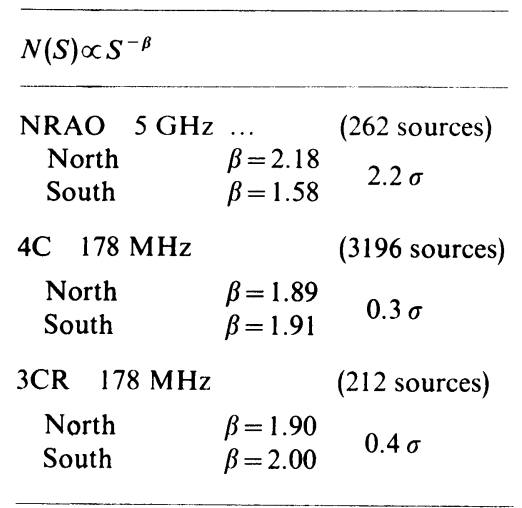

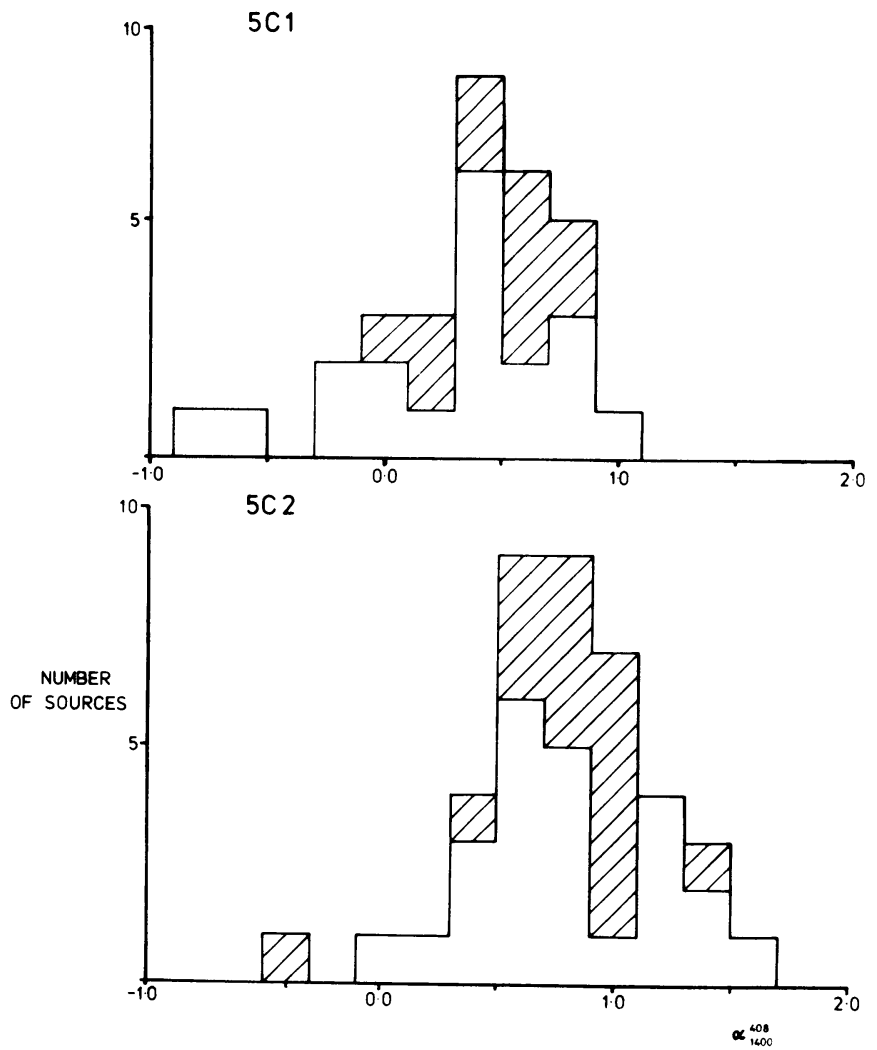

Fig. 9. Comparison of the spectral index distributions in the 5C 1 and 5C 2 areas (Maslowski, 1972). The shaded boxes indicate those sources which are blends of two or more $5 \mathrm{C}$ sources. 
and $5000 \mathrm{MHz}$ and the difference persists no matter which combination of data is considered. It is more difficult to estimate what significance should be attributed to the effect since the statistics are small and the principal uncertainty results from the errors in the measurement of individual spectra. A similar phenomenon has been discovered by Shimmins and Bolton (1974b) for the southern sky.

(vi) The Parkes $2700 \mathrm{MHz}$ survey is now complete over large regions of the southern sky and for a number of regions Shimmins and Bolton have evaluated the spectral index distribution between 2.7 and $5 \mathrm{GHz}$ for sources selected at $2.7 \mathrm{GHz}$. Shimmins and Bolton find that the fraction of sources with spectral indices smaller than 0.5 varies across the sky. Their results for 6 regions are shown in Table II. Shimmins

TABLE II

Parkes Surveys of spectral indices for Sources selected at $2700 \mathrm{MHz}$

(Shimmins and Bolton, 1974b)

6 independent regions have been surveyed to $0.35 \times 10^{-26} \mathrm{~W} \mathrm{~m}^{-2}$

$\mathrm{Hz}^{-1}$. The following variations in the percentage of sources with spectral indices $\alpha \leqslant 0.5$ are found

\begin{tabular}{ll}
$\begin{array}{l}\text { Total number of } \\
\text { sources in region }\end{array}$ & $\begin{array}{l}\text { Percentage of } \\
\text { sources with } \\
\alpha \leqslant 0.5\end{array}$ \\
\hline 180 & 25 \\
149 & 28.2 \\
193 & 26.9 \\
214 & 23.4 \\
121 & 32.2 \\
92 & 41.3 \\
\hline
\end{tabular}

and Bolton believe that the variations represent real fluctuations in the percentage of sources with flat spectra and that the scale of the fluctuations is $\sim 0.3 \mathrm{sr}$. It will be of great interest to discover whether the effect persists when the Parkes survey is complete.

I will return to the question of anisotropy in the last section.

\section{Interpretation}

I believe that one can take three different approaches to interpreting the counts.

(i) One can take the attitude that the redshifts of quasars are not cosmological, there must be some totally new physics which we do not know about, we cannot claim to know anything about the large scale structure of the Universe, in particular the Einstein-Friedmann models may be based on incorrect physics, and in principle 
the whole Universe might stop at a (cosmological) redshift of 0.3 . I will not discuss this approach further.

(ii) One can take the less extreme view that the redshifts of quasars are not wholly cosmological but that the basic structure of the conventional world models is correct. I suspect that one could demonstrate that the latter statement is a necessary consequence of the fact that the Universe is expanding locally and that the microwave background radiation is isotropic. In this view one can set lower limits to the true distances of the objects contributing to the source counts from the observed convergence of the source counts at low flux densities or from the integrated radio background emission. This argument was first presented by Ryle (1958) and a more recent version by Longair and Rees (1972). This argument also imposes severe constraints on those models of the distribution of radio sources in which the deficit of high flux density sources is attributed to a local hole in an otherwise uniform distribution.

If the convergence of the counts at low flux densities were attributed to a single luminosity class of radio source, the minimum distance of sources observed in high flux density surveys would be $z \approx 0.03$. If there is a dispersion in radio luminosity, this lower limit must be increased by the square root of the spread in radio luminosity. A conservative estimate would then suggest that typical radio sources would have to have redshifts greater than 0.3 . Thus in the local hole model, radio sources at high flux densities would have to have redshifts of at least 0.3 so that the hole extends to genuinely cosmological distances. In this picture we are therefore forced to adopt the view that the radio sources with which we are dealing form a genuinely distant cosmological population with the consequent necessity of strong evolution.

(iii) The third approach is to adopt the conventional view that the redshifts of the quasars are genuinely cosmological and then one must adopt a picture in which there is strong evolution of the radio source population. In this picture unidentified sources are assumed to be very distant galaxies or intrinsically faint quasars.

The evolutionary picture. First let us investigate the expected behaviour of the counts for world models in which radio sources are assumed to be uniformly distributed and in which we incorporate the known dispersion in luminosity of the sources contributing to the counts. The procedure adopted was to work out the differential source counts at $408 \mathrm{MHz}$ from the luminosity distribution of radio sources at high flux densities at $408 \mathrm{MHz}$ for different classes of source, such as identified galaxies with redshifts, unidentified sources assumed to have different cosmological redshifts, etc. The results are summarised in Figure 10. It is clear that the steepest slope we ever obtain has $\beta=1.25$ at high flux densities rather than the Euclidean prediction of 1.5. Even a source count having slope 1.5 is distinctly anomalous. A similar analysis for steady-state cosmology has been given by Schmidt (1972).

The types of evolutionary model required to reproduce the observed counts have been extensively treated in the literature and need not be discussed here (for survey; see Longair, 1971). Most of the previous analyses have been devoted to deriving suitable evolutionary models at a single frequency but it is now important to be able to reconcile all the counts at different frequencies within a single model. What 
we want to know is the generalised luminosity function

$$
\varrho(P, z, \text { spectrum })
$$

which describes the space distribution of all radio sources having different types of spectra and luminosities as a function of cosmic epoch or redshift. This is the function which we really want to know since it gives directly the relative populations of different types of source with epoch and can possibly give information about the epoch

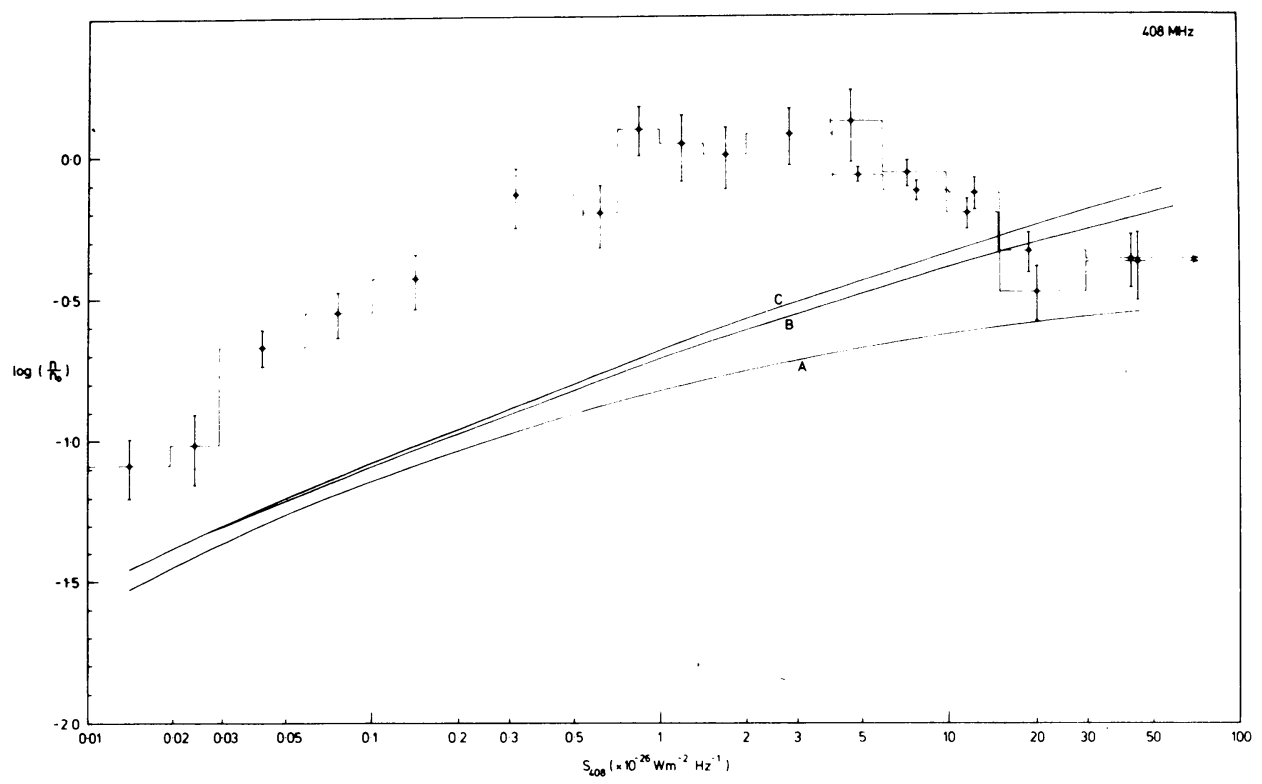

Fig. 10. Comparison of the expected differential counts of sources at $408 \mathrm{MHz}$ with the observed source count. The luminosity function was derived by selecting sources from $4.67 \mathrm{sr}$ of sky which had $S_{408} \geqslant$ $\gg 8 \times 10^{-26} \mathrm{~W} \mathrm{~m}^{-2} \mathrm{~Hz}^{-1}$. Curve (A) shows the expected count on the basis of the galaxies with measured redshifts in this sample. Curve (B) shows the expected count on the basis of all identified sources with estimates of their redshifts if necessary; for the quasars in this sample it is assumed that their redshifts are cosmological. Curve (C) shows the expected count for all sources following the procedure for curve (B) and assuming that all the unidentified sources have redshift 0.25 . The latter assumption is a conservative one and leads to the largest value of $\beta$ for the predicted count. These curves are correctly normalised to the observed number of sources (per sr) having $S_{408} \geqslant 8 \times 10^{-26} \mathrm{~W} \mathrm{~m}^{-2} \mathrm{~Hz}^{-1}$.

of quasar and galaxy formation. Fanaroff and Longair (1973) have made a first attempt at finding a suitable form for the function $\varrho(P, z, \alpha)$ and for illustrative purposes we assumed it to be factorisable at low frequencies, i.e. $\varrho(P, z, \alpha)=\varrho(P, z) \eta(\alpha)$ where $\eta(\alpha)$ is called the spectral index function. The procedure for working out counts and luminosity distributions at all other frequencies is described in our paper. These models predict a strong dependence of the fraction of sources with flat spectra on flux density at high frequencies as is illustrated in Figure 11. This type of dependence is found in the observations of Wall (1972) and Shimmins and Bolton (1974b). It has also been observed in the $5 \mathrm{GHz}$ observations of Pauliny-Toth and Kellermann 


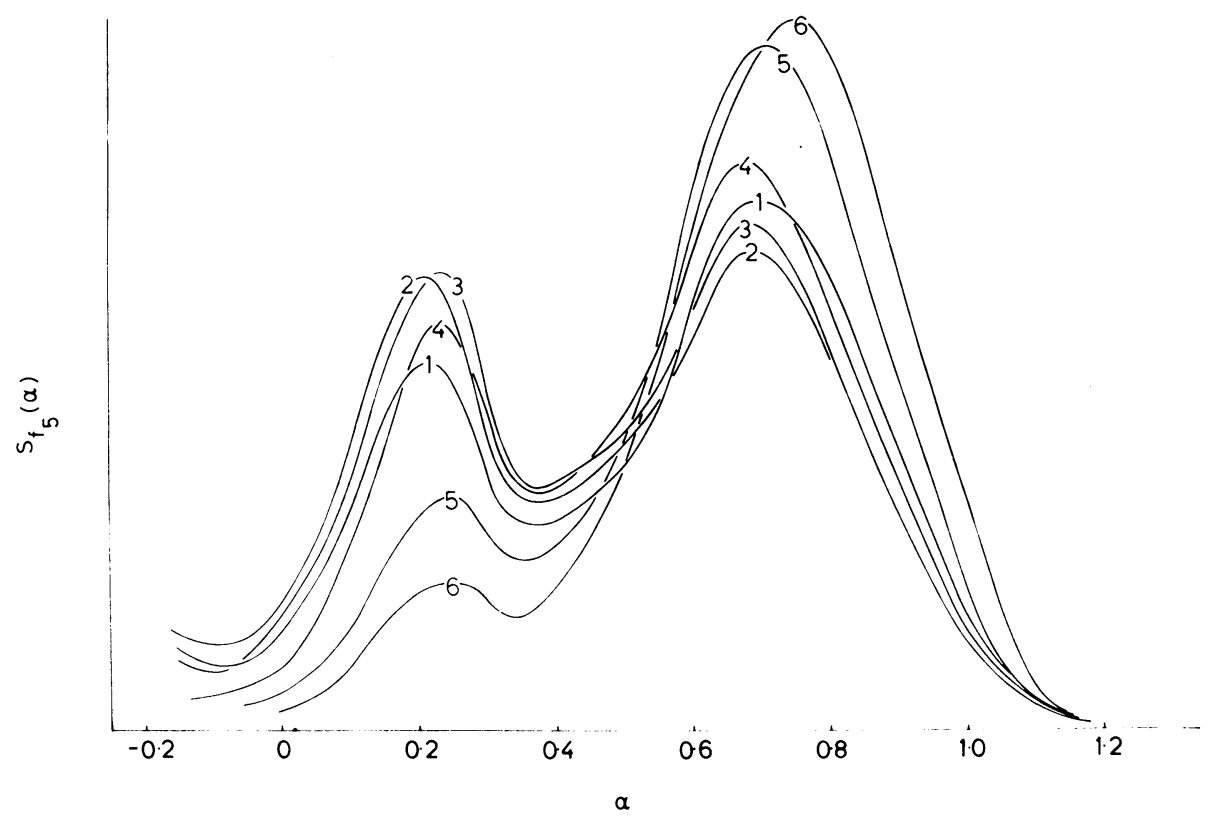

Fig. 11. The expected variation of the spectral index distribution with flux density at $5000 \mathrm{MHz}$ (Fanaroff and Longair, 1973). The curves numbered 1-6 refer to $S_{5000}=30,6,2,0.6,0.07,0.005 \times 10^{-26} \mathrm{~W} \mathrm{~m}^{-2} \mathrm{~Hz}^{-1}$.

(1972). Furthermore, we predict the distribution of radio sources in the radio luminosity - spectral index diagram for sources selected at $5 \mathrm{GHz}$. Our predictions are given in Table III and the observed diagram in Figure 12. It can be seen that the agreement is satisfactory.

A corollary to this result is that since the evolving component of the radio source population is assumed to be independent of the spectral index function at a low frequency, the evolving component has a broader dispersion in luminosity at high

TABLE III

A typical predicted distribution of spectral indices among sources of different luminosities in a survey at $5000 \mathrm{MHz}$ at flux density $S_{5000} \geqslant 2 \times 10^{-26} \mathrm{~W} \mathrm{~m}^{-2} \mathrm{~Hz}^{-1}$. The distribution is normalised to 100 sources. (Fanaroff and Longair, 1973)

\begin{tabular}{lrr}
\hline $\begin{array}{lr}\log P_{5000} \\
\left(P \text { in } \mathrm{W} \mathrm{Hz}^{-1} \mathrm{sr}^{-1}\right)\end{array}$ & $\alpha<0.5$ & $\alpha \geqslant 0.5$ \\
\hline $28-26$ & 44 & 21 \\
$26-24$ & 5 & 23 \\
$24-22$ & 1 & 6 \\
\hline
\end{tabular}




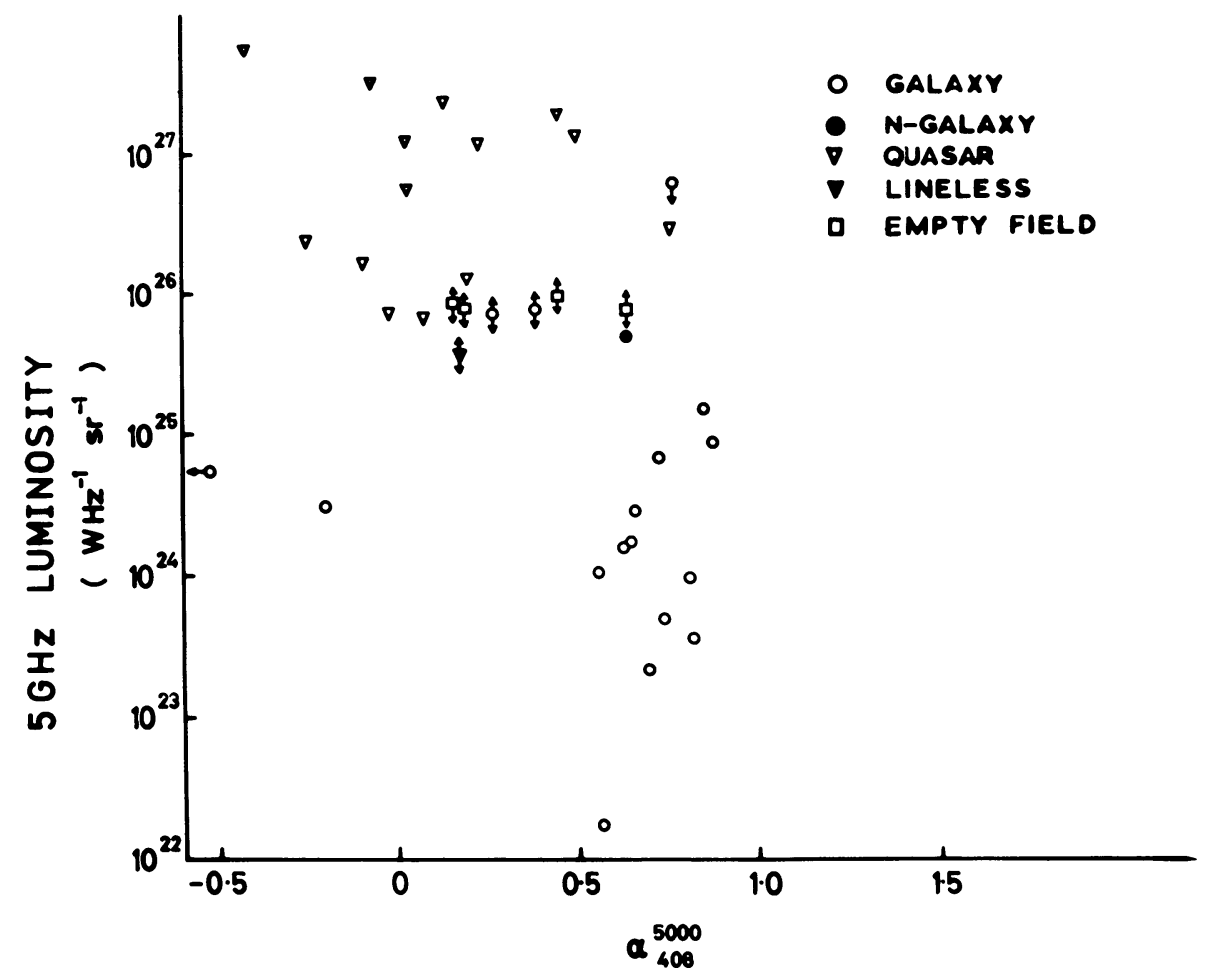

Fig. 12. The observed radio luminosity - spectral index diagram for sources selected at $5000 \mathrm{MHz}$ with $S_{5000} \geqslant 2 \times 10^{-26} \mathrm{~W} \mathrm{~m}^{-2} \mathrm{~Hz}^{-1}$. The data were chosen from the catalogues of Pauliny-Toth et al. (1972) and Pauliny-Toth and Kellermann (1972).

frequencies because there is a strong induced correlation between high radio luminosity and low spectral index. This means that the maximum in the differential source counts expressed as $n / n_{0}$ is expected to be much broader at high frequencies. This is illustrated in Figure 13 for the integral counts. In the existing surveys at high frequencies we observe predominantly the broad maximum of the counts rather than the region with slope 1.8 .

Some of these results can be recovered by simple convolution of the observed source counts with a suitable spectral index function without ever introducing any dependence of the properties of the sources on luminosity or redshift. In this way the dependence of the fraction of flat spectrum sources upon flux density can be recovered (as in Figure 11) and the high and low frequency counts can be shown to be consistent. However, it is impossible to incorporate the redshift information into such analyses. The observations are now of such a quality that it is important to investigate how well the evolutionary models can account for all the existing data. We have demonstrated that a simple modification of the evolutionary world models - the introduction of the spectral index function - enables us to account for all the observations. Further analyses should enable us to estimate the ability of the source counting procedure in determining the epoch of quasar and galaxy formation. 


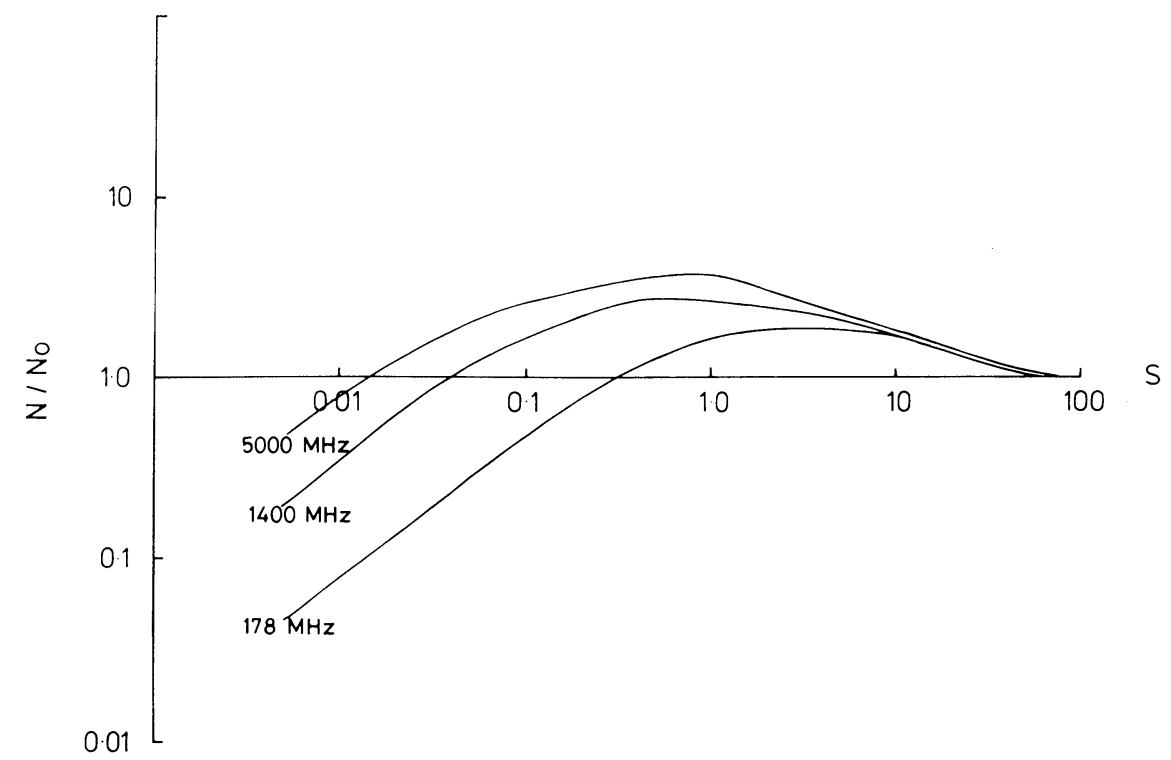

Fig. 13. The integral source counts expected at different frequencies on the basis of the models described in the text (see also Fanaroff and Longair, 1973).

\section{Conclusions}

Models of the distribution of radio sources which include strong evolution of the radio source population with cosmological epoch can satisfactorily account for all the present observations. Only if we are prepared to adopt a radical stance in which it is believed that we do not know anything about the physics applicable to the Universe on the largest scale can we escape this evolutionary picture. In my view there is no compelling evidence to support this view.

What attitude should we adopt to the anisotropies discussed in Section 3 ? The most urgent requirement is a very careful reappraisal of all these results and the extension of the observations to cover as much of the sky as is practicable. At the present time we are re-analysing the $5 \mathrm{C} 2,3$ and 4 surveys and we are in the process of performing three further surveys, 5C 5,6 and 7 to look for anisotropies.

If the anisotropies are substantiated by future work, can they readily be incorporated into the overall picture? They would seem to be readily explicable in the strong evolutionary models since radio sources are observed at effectively a single large redshift. To put it another way, because of the strong evolution the interval of cosmic time over which one sees the bulk of radio sources contributing to the excess is much smaller than in a uniform model. The anisotropies might therefore reflect fluctuations associated with the largest scale cosmic structures at their time of formation. These would be expected to be most marked around the maximum in the differential source counts as expressed by $n / n_{0}$. This question merits much further study.

It should also be remembered that eventually we should detect the superclustering 
of distant radio sources associated with the equivalent of Abell clusters. It is expected that this should become observable just about the sensitivity limits of the deepest available surveys - i.e. about 1 to $10 \times 10^{-29} \mathrm{~W} \mathrm{~m}^{-2} \mathrm{~Hz}^{-1}$.

My final platitude is commonplace - there remains a great deal to be done.

\section{Acknowledgements}

In preparing the material of this report, I have been greatly assisted by my colleagues, in particular Tony Hooley and Tim Pearson with whom a more detailed account of this work will be published elsewhere. I am also very grateful to John Bolton and John Shimmins for allowing me to present the results of the Parkes $2700 \mathrm{MHz}$ survey before publication and to Richard Porcas and Dennis Walsh for permission to quote their unpublished counts at $2700 \mathrm{MHz}$. To all of these colleagues I am grateful for stimulating discussions.

\section{References}

Bennett, A. S.: 1962, Mem. Roy. Astron. Soc. 67, 163.

Bolton, J. G. : 1971, 'Extragalactic Radio Phenomena', invited paper at 12th International Conference on Cosmic Rays, Hobart. p. 111.

Bridle, A. H., Davis, M. M., Fomalont, E. B., and Lequeux, J.: 1972, Nature 235, 123.

Caswell, J. L. and Crowther, J. H.: 1969, Monthly Notices Roy. Astron. Soc. 145, 191.

Davis, M. M.: 1971, Astron. J. 76, 980.

Davis, M. M.: 1974, Astron. J., in press.

Ekers, J. (ed.): 1969, Australian J. Phys. Astrophys. Suppl., No. 7.

Fanaroff, B. L. and Longair, M. S. : 1973, Monthly Notices Roy. Astron. Soc. 161, 393.

Flin, P., Machalski, J., Maslowski, J., Urbanik, M., Zieba, A., and Zieba, S.: 1974, this volume p. 121. Gower, J. F. R.: 1966, Monthly Notices Roy. Astron. Soc. 133, 151.

Hewish, A.: 1961, Monthly Notices Roy. Astron. Soc. 123, 167.

Hinder, R. A. and Branson, N. J. B. A. : 1969, Observatory 89, 178.

Holden, D. J.: 1966, Monthly Notices Roy. Astron. Soc. 133, 225.

Hughes, R. G. and Longair, M. S. : 1967, Monthly Notices Roy. Astron. Soc. 135, 131.

Katgert, P., Katgert-Merkelijn, J. K., Le Poole, R. S., and van der Laan, H.: 1973, Astron. Astrophys. 23, 171.

Longair, M..S.: 1971, Rep. Progr. Phys. 34, 1125.

Longair, M. S. and Rees, M. J.: 1972, Comments Astrophys. Space Phys. 4, 79.

Maslowski, J.: 1972, Astron. Astrophys. 16, 197.

Maslowski, J.: 1973a, Acta Astron. 22, 227.

Maslowski, J.: 1973b, Astron. Astrophys. 26, 343.

Mills, B. Y., Davies, I. M., and Robertson, J. G.: 1973, Australian J. Phys. 26, 417.

Pauliny-Toth, I. I. K. and Kellermann, K. I.: 1972, Astron. J. 77, 560.

Pauliny-Toth, I. I. K., Kellermann, K. I., Davis, M. M., Fomalont, E. B., and Shaffer, D.: 1972, Astron. J. 77, 265.

Pearson, T. J.: 1974, Monthly Notices Roy. Astron. Soc. 166, 249.

Pooley, G. G. and Ryle, M.: 1968, Monthly Notices Roy. Astron. Soc. 139, 515.

Robertson, J. G. : 1973, Australian J. Phys. 26, 403.

Ryle, M.: 1958, Proc. Roy. Soc. A248, 289.

Ryle, M. and Neville, A. C.: 1962, Monthly Notices Roy. Astron. Soc. 125, 39.

Schmidt, M.: 1972, Nature 240, 399.

Shimmins, A. J. and Bolton, J. G. : 1974a, 'Counts of Radio Sources at $5009 \mathrm{MHz}$ ' in preparation. 
Shimmins, A. J. and Bolton, J. G. : 1974b, 'Distributions of Effective Spectral Indices Between 2700 and $5009 \mathrm{MHz}$ ' in preparation.

Wall, J. V.: 1972, Australian J. Phys. Astrophys. Suppl., No. 24.

Yahil, A.: 1972, Astrophys. J. 178, 45.

\section{DISCUSSION}

Zel'dovich: It is possible to determine whether there is a cut-off in the distribution of radio sources at large redshifts from the counts of radio sources?

Longair: I believe it is impossible at present to say whether or not there is an abrupt cut-off to the distribution of radio sources at large redshifts. With improved statistics in the region of convergence of the counts, it may be possible to say something about this question. I believe that the most direct evidence concerning whether or not there is such a cut-off will come from the redshift distribution for quasars.

Zel'dovich: Can Dr Schmidt comment on the evidence from the redshift distribution of quasars?

Schmidt: From present quasar evidence it appears that their comoving density increases little or not at all beyond $z=2.5$. This would allow exponential evolution of the form $10^{-11 t /(\text { age })}$ but not unlimited evolution of the form $(1+z)^{6}$.

Rowan-Robinson: I would like to suggest an alternative approach to source count anisotropies. These appear only at high frequencies where a far higher proportion of the sources have flat spectra than at low frequencies. The majority of these are probably flat-spectra, compact quasars which I have suggested are comparatively weak, nearby objects similar to Seyfert nuclei. The anisotropies would then be due to inhomogeneities on a reasonable scale, whereas if the sources are at a redshift of 2 we are talking about inhomogeneities several thousands of $\mathrm{Mpc}$ in size if there is a real difference between the northern and southern galactic hemispheres.

Longair: My principal worry about the reported anisotropy between the northern and southern galactic hemisphere is that it is based upon a posteriori statistics, i.e. you see something anomalous and then ask how likely it is to occur. If the effect had been noticed in all surveys and was significant at the 5 or $6 \sigma$ confidence level, then one could not have ignored the effect. However, this is not the case. Fortunately, it will soon be possible to repeat the statistics with much larger numbers using the Parkes $2700 \mathrm{MHz}$ survey.

\section{Some Critical Remarks about Source Counts and Cosmology}

van Hoerner: Most authors who have compared source counts with cosmological models agree that evolution is needed and is more important than the choice of the right world model. But there is much disagreement about all of the details: whether density evolution is sufficient, or luminosity evolution is needed in addition, (or instead of); whether all sources evolve, or only the bright ones, or only the quasars; and so on.

A general investigation into the role played by the radio luminosity function $\Phi(L)$ gave four results. First, there is a 'critical shape', $\Phi \propto L^{-5 / 2}$; unfortunately, the data follow this critical shape, within their range of uncertainty, over four powers of ten in $L$. The disagreeing results about evolution can be obtained by using slightly different luminosity functions, on one or the other side of the critical one. The results concerning the details of the evolution thus are dominated by the choice of the right luminosity function.

Second, the normalized differential counts $n_{n}(S)$ consist of three parts : a maximum or rather flat top at intermediate flux densities $S_{m}$, a drop at the bright end, and a steep decrease to faint flux densities; these parts have different meanings. The drop needs evolution such that not the density but the integral over $\Phi_{n}=L^{5 / 2} \Phi$ was higher in the past; the location $S_{m}$ of the maximum depends on the location $L_{m}$ of the maximum of $\Phi_{n}$ and on the redshift cutoff, and the broad maximum of the counts needs a broad maximum of $\Phi_{n}$ and /or a more gradual redshift cutoff. But the decrease to faint flux densities depends more on the luminosity function than on cosmology. Unfortunately, future observational progress will be for faint sources only.

Third, not only is the expected Hubble relation $\left(z \propto S^{-1 / 2}\right)$ blurred by too much scatter; in addition, we find that even the expected slope of the Hubble relation may have any value (plus, minus, zero) depending on the luminosity function and evolution. The most realistic assumptions yield a negative slope only for the brightest flux densities, but a small positive slope for the faint ones (reverse Hubble relation : faint sources are nearby). Whenever the slope is close to zero, source counts have no bearing on cosmology since the 
average flux density then is no distance indicator. This shows the need for including angular structure and spectra in addition to just flux densities and numbers.

Fourth, after having finished the evaluation of a source count, one must calculate $z(S)$ for the bright sources and compare with observations. Several otherwise good evolutionary models had to be rejected as a result of this redshift test. One must find a compromise for fitting both the counts and the redshifts.

Longair: I think most workers agree about the gross features of the evolution necessary to explain the source counts. The questions of detail to which you refer cannot be answered unambiguously at the present time. I dislike the distinction which is drawn between the extreme cases of luminosity and density evolution since what we are really trying to find out is the way in which the luminosity function changes with cosmological epoch. These changes in the luminosity function can be interpreted as luminosity evolution or density evolution or some intermediate combination of the two. The only unambiguous way of determining how the properties of different classes of source evolve with epoch is by finding some 'label' which defines objects of a particular intrinsic type. There has been little success in finding such labels to date.

Concerning the 'critical' shape of the luminosity function, the shape of this function does not affect the overall predictions of the source counts in models which do not incorporate cosmological evolution the integral count always has slope less than 1.5. The shape is 'critical' in the sense that at the very highest flux densities where the first members of each luminosity class appear, they all appear first at about the same flux density for this form of luminosity function, i.e. they result in a flat luminosity distribution $n(P, S)$. It might be thought that at such small source densities the counts might reflect the luminosity function rather than the space distribution of sources. However, if the sources are randomly distributed in space, the predicted count has slope less than 1.5 even at very small source densities (I am grateful to Dr John Shimmins for discussion of this point).

Tammann: You mentioned briefly the existence of superclustering as a matter of course. Do you rely here on the optical evidence, as described so impressively by Dr Abell this afternoon or do you have additional evidence?

Longair : My remarks were theoretical ones based upon the known properties of the radio sources which appear in Abell's clusters and the fact that it is now generally agreed that there is clustering of Abell's clusters themselves. On this basis one expects to encounter clustering of faint radio sources at about $S_{408}=10^{-29} \mathrm{~W} \mathrm{~m}^{-2} \mathrm{~Hz}^{-1}$. Of course extending counts to $S_{408} \approx 10^{-31} \mathrm{~W} \mathrm{~m}^{-2} \mathrm{~Hz}^{-1}$ should enable us to detect ordinary clusters of galaxies at cosmologically interesting distances! 